tural Materialism“ aber jedem empfohlen werden, der sich umfassender mit Raymond Williams bzw. den Cultural Studies und ihrem weiteren Theoriekontext befassen möchte. Zwar ist Raymond Williams sicherlich nicht der „Gründungsvater" der Cultural Studies und zwischen seinem späteren Werk und der weiteren Entwicklung der Cultural Studies bestehen insofern Differenzen, als er die Wende von vielen Vertreterinnen und Vertretern der Cultural Studies hin zum Poststrukturalismus nicht nachvollzog. Jedoch haben sowohl Williams' Veröffentlichungen - insbesondere „Culture and Society" und "The Long Revolution" - als auch sein Engagement als kritischer Intellektueller nachhaltig die Entwicklung der Cultural Studies international beeinflusst. Die Lektüre von Higgins' nicht ganz lückenloser, intellektueller Biografie von Raymond Williams trägt sicherlich dazu bei, auch gegenwärtige Diskussionen in den Cultural Studies nachvollziehen zu können. Vor allem aber hilft sie, den Projektcharakter von Cultural Studies verstehen zu können, für die ein ähnlicher Anti-Dogmatismus kennzeichnend ist wie für den kulturellen Materialismus von Raymond Williams. Befragt darauf, wie sein Werk im Kontext einzelner Schulen einzuordnen wäre, antwortete Raymond Williams 1971: „If you are not in a church, your are not worried about heresies" (zitiert nach Higgins 1999, S. 101).

\section{Andreas Hepp}

\section{John Hartley}

\section{The Uses of Television}

London: Routledge 1999. - 246 S.

\section{ISBN 0-415-08509-8}

„Go on! And watch a lot of television!“ Man stelle sich diese Aufforderung in einer kommunikationswissenschaftlichen Einführung vor, die sich an ein Publikum richtet, das schon immer einmal wissen wollte, was es denn mit diesem Medium eigentlich auf sich hat. Zwar steht dieser Satz nicht am Ende des Textteils, sondern in einem Teil des Anhangs, der Anleitungen für „Do-it-Yourself TV studies“ enthält. Aber nach der anstrengenden Lektüre eines eigenwilligen und gelegentlich provozierenden Textes muss man wohl zu dem Ergebnis kommen: Es ist dem Autor ein ernstes Anliegen, ein „desire“.
Als Hartley dieses Buch verfasste, lag seine Wirkungsstätte noch in Wales an der Cardiff University, mittlerweile ist er ein „new australian" und wirkt an der Queensland University of Technology. „Uses of Television“ reiht sich ein in eine Vielzahl von Arbeiten zur Populärkultur, von denen hier beispielhaft nur "Reading Television“ (1978, mit John Fiske) und "The Politics of Pictures“ (1992) genannt werden sollen.

Das Buch ist eine Mischung aus Appell, Vision, Idee und Kritik, ein im Wesentlichen auf historische Entwicklungen der insgesamt noch jungen Fernsehgeschichte Bezug nehmendes Argumentieren für Sichtweisen, die einen unbefangeneren und positiveren Umgang mit dem Medium rechtfertigen sollen. Zugleich eine wohlwollend kritische Erinnerung an Richard Hoggarts "The Uses of Literacy“ (1957/1958), eine Untersuchung des Einflusses der Massenmedien auf Werthaltungen und Mentalität der britischen Arbeiterklasse mit Pionierfunktion für die Cultural Studies.

Vieles wiederholt sich auf den ca. 200 Seiten. Und eigentlich beginnt sich ein Lesevergnügen meistens dort einzustellen, wo der Verfasser das undurchsichtige Feld theoretischer Kontroversen um Kultur und Gesellschaft verlässt und die Anfänge des Fernsehens (insbesondere in Großbritannien) mit amüsanten Episoden anschaulich macht. Dazu gehört beispielsweise die Beschreibung der hohen Wertschätzung des Radios seitens der Verantwortlichen der BBC und die daraus resultierende nachrangige $\mathrm{Be}-$ handlung des Fernsehens. Noch 1951 äußerte sich Maurice Gorham, ein ehemaliger Leiter des BBC Television Service: „It is surely obvious that the television medium [...] is the ideal vehicle for the radio newspaper that will undoubtedly come in time, even here." (S. 89) Die Abdankung Edwards VIII. im Jahr 1936 war noch ein „radio event“. Erst die Krönung von Elisabeth II. im Jahr 1953 machte das Fernsehen zu einer „popular cultural form“ (S. 90). Wenig später, im Jahr 1955, war es eine Entscheidung der britischen Regierung, die dem Fernsehverständnis der BBC durch die Einführung von Independent Television (ITV) intramediäre Konkurrenz bescherte (ein „element of competition“), die zuvor doch vorwiegend intermediär wahrgenommen wurde. Ebenso fand damit die amerikanische Variante des Fernsehens Verbreitung. Hartley präsentiert zu diesen Ereignissen immer auch passen- 
de Bilder bzw. Werbeanzeigen. Die Geburt der „choice-oisie“ im Jahr 1955 führte beispielsweise zu einer Karikatur (ein Fernsehbildschirm zeigt das Gesicht eines Mannes, ein weiterer das einer sprechenden Frau, eine Frau als Zuschauerin) mit folgendem Text: „It's hard to choose between rival attractions, but we sort out guinea-winning letters." (S. 90) Amüsant auch die Ausführungen zu frühen Versuchen, die Mängel von Kameras und Übertragungstechniken $\mathrm{zu}$ vertuschen. Während eine von Baird entwickelte Kamera nicht nur ein schwerfälliges Instrument darstellte, sondern aufgrund der verwandten Chemikalien die Kameramänner regelmäßig in Lebensgefahr brachte, musste der Qualität des Bildes im Falle des so genannten EMI-Systems wie folgt abgeholfen werden: „The bizarre colours of make-up that presenters had to wear in order to look ,natural ' for the EMI system (blue and russet faces scanned better than pinkish ones) seemed a smaller price to pay" (S. 59).

Aber das alles ist sicher nicht der Kern von „Uses of Television“, wenngleich gerade diese Nebensächlichkeiten wahrscheinlich länger im Gedächtnis bleiben. Schon auf den ersten Seiten werden Marksteine gesetzt, die einen überraschen, aber natürlich auch gespannt auf die Antwort machen: Dazu gehört an erster Stelle: „I am going to suggest that the uses of television are best understood by means of the concept of TRANSMODERN TEACHING" (S. 26). Sodann die Feststellung: „I am proposing that the best model of cross-demographic communication currently to hand is television" (S. 32). Schließlich die unter der Überschrift „Democratainment“ skizzierte Kategorie „media citizenship“. Die Kritik an einzelnen Autoren (z. B. an Raymond Williams und Stuart Hall), die in nahezu jedem der insgesamt 15 Kapitel eine Rolle spielt, ist sicherlich Teil einer internen Debatte um Cultural Studies. Hier interessieren die Kernaussagen, nicht die Kontroversen unterschiedlichster Art.

Was soll der Begriff bzw. das Konzept „transmodern teaching" verdeutlichen? Kein Lernen, kein Sammeln von Erfahrungen, das sich an vorgegebenen Curricula orientiert, formale Kriterien aufweist und nur diesen Geltung zuschreibt. Fernsehen als Medium und als Aktivität ist nicht vergleichbar mit dem Besuch einer Schule. Wen mag das überraschen? $\mathrm{Zu}-$ gleich ist die zugrunde liegende Argumentation nicht unbekannt, erfährt aber eine andere Ein- bettung. Das Fernsehen ist vormodern, modern und postmodern zugleich. Es vereint Phänomene, die unvereinbar erscheinen und bereitet damit neue Erfahrungen. Es greift zurück auf alles, was schon einmal da gewesen ist und verwertet bzw. instrumentalisiert es für eine Vielzahl von Zwecken, was wohl einen Teil seiner Unbestimmtheit begründet. Es greift zurück auf mündliche Formen der Kommunikation (ein „pre-modern mode of communication“, S. 41) und übernimmt in einer säkularisierten Form die soziale Funktion der mittelalterlichen katholischen Kirche (übrigens ein Vergleich, den auch die Annenberg School of Communication um George Gerbner gerne heranzieht). Es ist eine andere Form des "teaching“, eine Form, die nicht auf Wissen Bezug nimmt, das in Büchern steht. Es ist ein Medium, das Differenzen integriert und auf diesem Weg alte Unterscheidungen und Exklusionen übergeht: „If TV is teaching $[\ldots]$ it is teaching audiences about cultural distinction, the expansion of difference, and the segmentation of both cultural productivity itself and of the audience for it" (S. 45).

Diese Perspektive konnte sich in der bisherigen Tradition der „TV studies“ wenig entfalten, weil lange Zeit eine Fernsehkritik dominierte, die sich gar nicht mit dem Fernsehen selbst beschäftigte (Missachtung der „textual tradition"), ihm aber dennoch die Ursache für eine Vielzahl von „external factors“ (S. 65) zuschrieb. Die moralisierenden Vorgaben dieser ebenfalls externen Beobachter haben schon ihre Wirkung entfalten können, bevor das Medium selbst überhaupt eingeführt war: „In this context, television's reputation was established before it went on air" (S. 69). Und natürlich wurden die Tugenden und Geschmäcker der Kritiker ohne Konsultation der Nutzerschaften als gut und nachahmenswert empfohlen. Die Mediengeschichte kennt aber in dieser Hinsicht wohl kaum eine Ausnahme, so dass der Diskurs über das Fernsehen insofern keine Sonderstellung beanspruchen kann. Der Film bzw. das Kino musste den Vergleich mit dem Theater erdulden, das geschriebene Wort den Vergleich mit dem Dialog usw.

Aber für Hartley ist es auch eine Angst vor Demokratisierung gewesen, die dem Fernsehen die Rolle des „evil“"verschaffte. In den geäußerten Befürchtungen realisieren sich Gesellschafts- und Erziehungsideale, Vorstellungen über Ästhetik, im weiteren Sinne Grundüber- 
zeugungen bezüglich einer guten Ordnung, die eine Elite definiert und verteidigt. Lässt man einmal die vielen Seitenhiebe auf die weiteren Beteiligten - die Sozialwissenschaften, die „psycho-sciences“ und "Mass-comm“-Forscher (S. 128) - unkommentiert, dann spiegelt sich in dieser "pathologization of everyday life"(S. 104) eine tief sitzende Abneigung gegen Populärkultur. Nach Hartley hat auch die amerikanische Fernsehforschung (die von ihm mehrheitlich als management science wahrgenommen wird) ihrem Forschungsgegenstand wenig Positives abgewinnen können. Immerhin meint er damit auch die Forschungsarbeiten der 1970er Jahre: „Reading this early American research on television, there is little sense that television (unlike film) was an enjoyable medium to watch (for the researchers, especially), nor that the ,mass' audience's motivations for watching it might be positive, nor that its social, psychological or political impact was anything other than pathological“ (S. 131).

Insgesamt kann Hartley nur wenige Wissenschaftler ausfindig machen, die sich gegen eine „fear"-Tradition und für eine „Desire“-Perspektive entschieden haben (selbst innerhalb der Cultural Studies existiere ein "passionate dislike for post-political fun“, S. 122) oder zumindest unvoreingenommen das Medium zunächst einmal als das nehmen, was es ist: Marshall McLuhan, Umberto Eco, Horace Newcomb. Letzterer hat alleine durch die Herausgabe einer 2000 Seiten starken Encyclopedia of Television unter Beweis gestellt, dass er kaum einer „fear school“ zugeordnet werden möchte, Eco steht für die Begründung einer semiotischen Analyse des Mediums und McLuhan für eine historische Einordnung desselben unter Berücksichtigung der Spezifika der Technologie. Insbesondere aber sei er der Erste gewesen, der die Auffassung vertrat, „that the prospects for cross-demographic communication among strangers were enhanced rather than damaged by television" (S. 134). Das wird Forscher, die sich seit vielen Jahren mit interkultureller Kommunikation beschäftigen, neugierig machen. An dieser Stelle sei ein Hinweis auf Joshua Meyrowitz erlaubt, der sich in seinen Arbeiten ebenfalls auf McLuhan beruft, dessen Beobachtungen aber in ihren Konsequenzen für Zeit- und Raumwahrnehmung, für Sozialisation und Orientierung in einer neuen „Erfahrungswelt" diskutiert. Dabei weist Hartley unter Rückgriff auf Hoggart selbst darauf hin, dass diese Erfahrungswelten aufgrund einer besonderen Sender-Empfänger-Beziehung eine bestimmte Struktur aufweisen. Der „effect of the audience on television" (S. 144) liegt in der Antizipation von Zuschauererwartungen durch die Programmmacher. Die Folge ist eine Nivellierung auf Durchschnittliches, das Vermeiden von Risiken, kurzum: die viel kritisierte Orientierung am Massengeschmack bzw. das Verharren an der Oberfläche und eine fehlende Tiefe der Erörterung. Die zur Illustration verwandten Werbeanzeigen zeigen sogar durchweg einen leeren Bildschirm. Was konkret gesehen wird, scheint also schon in den 1950er Jahren offen gewesen zu sein (vgl. die Abbildungen auf S. 146f.) Dennoch wird im Falle Hartleys die Entscheidung für eine „Desire“Perspektive zugleich $\mathrm{zu}$ einem euphorischen Appell und mündet in ein Szenario, das den Namen „Democratainment“ erhält.

Das Fernsehen wird zu einer unerschöpflichen Ressource für Meinungen, Moden, Trends, Lebensformen, Weisheiten, Alltagsgeschichten, Banalitäten, Kuriositäten, Nachrichten ... - eine endlose Liste. Was einem vor Jahr und Tag noch fremd erschien, wird nun in ein „neighbourliness“-Schema eingebettet, obwohl es in geographischer Hinsicht entgegengesetzte Dimensionen erreicht, z. B. „world's funniest home-video clips“ (S. 160). Diese Geschmacksnähe trotz Distanz ist nur ein Beispiel für ein Phänomen, dem Hartley den Namen „media citizenship“ gibt. Hier verwirklicht sich die Wahrnehmung von Differenz auf allen Ebenen. Neben die ortsgebundene Wahrnehmung und das an Orte gebundene Leben treten die "citizens of media“, die in dieser "mediasphere“ einer "semiosphere“ begegnen, in der natürlich auch Protagonisten wie Benetton, Coca-Cola und IBM den Ton mit angeben. Der Zutritt zu dieser Arena und den dortigen Angeboten ist nicht reglementiert. Paradigmatisch hierfür mag die Aufforderung von Microsoft sein: „where do you want to go today?" (S. 162). Dieses "do-it-yourself-citizenship“ wird durch das Individualisierungspotenzial neuer Informations- und Kommunikationstechnologien noch forciert und erweitert, bleibt aber dem Medium Fernsehen treu. Dieses Vielfaltscredo ist alles andere als unbekannt. Informationen, Informationen - man muss sie nur wahrnehmen.

Dadurch geraten natürlich klassische Integrationsmedien (public broadcasting) unter 
Druck. In dieser Phase der gesellschaftlichen Entwicklung findet auch das Fernsehen wohl endlich den Platz, der ihm zusteht: „It can stimulate the desire for freedom, comfort, politics, culture. That's what it has been ,for'. Those are the ,uses of television' (S. 188). Jede Spiegelbild-Metapher (Fernsehwirklichkeit und soziale Wirklichkeit) muss hier verblassen. Ausgerechnet das Fernsehen scheint plötzlich frei von Stereotypen und anderen Verkürzungen zu sein - eine ungewöhnliche Untermauerung der Komplementärthese. Jenseits der Techniken des „formal schooling“ entsteht auf diesem Weg wohl eine Gesellschaft von Autodidakten, die sich gerne etwas erzählen lassen, z. B. von Clarissa Darling in der Sendung „Clarissa explains it al“: „It is not serious, deep or ,critical' television, and may present to the new viewer as banal and ordinary, but of course that's the point" (S. 183). Ein Plädoyer für das Alltägliche. Das Fernsehen wird endgültig zum Medium des „intelligent layman“ (S. 29).

Dann ist da noch die Sache mit dem Kühlschrank. Mag sein, dass "the fridge " maßgeblichen Anteil an der Verhäuslichung des Lebens, insbesondere der britischen Arbeiterklasse, genommen (vgl. S. 99ff.) und eine Kausalkette in Gang gesetzt hat, die vielleicht die folgende Reihenfolge aufweist: Kühlschrank $\rightarrow$ Fernsehen $\rightarrow$ Werbung $\rightarrow$ Supermarkt $\rightarrow$ Auto (Mobilität) $\rightarrow$ Kühlschrank $\rightarrow$ Fernsehen $\rightarrow$ Werbung $\rightarrow$ Supermarkt $\rightarrow$ Kühlschrank $\rightarrow$ usw. Konsequenterweise bemerkt der Autor: „As far as I know there's no climate of cultural criticism directed at the fridge" (S. 104).

Wenn doch alles so einfach wäre und sich den Gegensätzen fügen würde, die Hartley so sehr mag: John Fiske vs. George Gerbner, „TV studies“" vs. „mass-comm“, „statistical sampling“" vs. „critical reading“, und schließlich: „Routledge" vs. Sage. Da fällt einem am Ende doch noch ein bekannter Appell aus der "Semiosphäre" ein: „Wer wird denn gleich in die Luft gehen?".

Michael Jäckel

\section{Philomen Schönhagen}

\section{Unparteilichkeit im Journalismus}

Tradition einer Qualitätsnorm

Tübingen: Max Niemeyer Verlag 1998. - 331 S.

(Medien in Forschung + Unterricht, Serie A; 46)

\section{ISBN 3-484-34046-0}

Begriffsanalysen sind unmodern geworden. $\mathrm{Zu}$ Unrecht, wie die von Hans Wagner betreute Untersuchung von Philomen Schönhagen zeigt. Die Verfasserin beginnt ihre Studie nach einem knappen, aber kenntnisreichen Überblick über den Forschungsstand mit einer Beschreibung des Selbstverständnisses von deutschen Publizisten vom 17. bis 19. Jahrhundert. Ihre Quellen sind vor allem pressehistorische Darstellungen. Gestützt auf zahlreiche Belege zeigt Schönhagen, dass Unparteilichkeit von Beginn der deutschen Pressegeschichte in verschiedenen sprachlichen Wendungen ein wesentliches Element des Selbstverständnisses der Berichterstatter bildete. Als systematischen Ertrag arbeitet die Verfasserin fünf Facetten des Begriffs der Unparteilichkeit heraus: (1) Prüfung der Quellen; (2) getreue Mitteilung der Sichtweisen; (3) Offenheit für verschiedene Sichtweisen; (4) Neutralität der Vermittlung und (5) Trennung von Bericht und Meinung (S. $50 \mathrm{f}$ ). Ist schon dieser Befund überraschend und seine Herleitung lesenswert, so gewinnt die Darstellung noch durch einen Exkurs über die Geschichte der Objektivitätsnorm in den USA an Plastizität. Schönhagen zeigt, gestützt auf zahlreiche amerikanische Pressehistoriker, daß die dortige Presse phasenweise erheblich parteiischer war, als man hierzulande meist vermutet.

Gestützt auf ihre Literaturstudie untersucht die Verfasserin eine (nicht repräsentative) Stichprobe von 1.500 Heimatzeitungen aus dem 18. und 19. Jahrhundert. Dabei handelt es sich - vorgegeben durch die Archivbestände der Universität München - „um Jubiläumsund Festnummern sowie Verlagsfestschriften “ (S. 14). Ihr Ziel besteht darin, aus den Primärquellen Belege für die Sachdarstellung der Sekundärliteratur zu finden. Dabei orientiert sie sich an den oben erwähnten Facetten der Unparteilichkeit. Für deren Relevanz findet sie in den Selbstdarstellungen der Blätter zahlreiche Beispiele. Zwar weist die Verfasserin selbst darauf hin, daß die "Jubelbeiträge" in den Fest- 